\title{
Guided Wave Arrays for High Resolution Inspection
}

\author{
Alexander VELICHKO, Paul WILCOX, Department of Mechanical Engineering, \\ University of Bristol, Bristol, UK
}

\begin{abstract}
The paper describes a general approach for processing data from a guided wave transducer array. The technique is based on phased addition in the frequency domain and can be applied to any array geometry and any types of array elements. The problem of finding optimal phased shifts, which allow the best resolution to be achieved with the minimum number of array elements, is investigated. Different algorithms are applied to modelling and experimental data and the results compared. Generalization for the case of multimode media is suggested.
\end{abstract}

\section{Introduction}

Ultrasonic guided waves are widely used in many areas of nondestructive evaluation [1-4]. In this paper the problem of synthesizing a high resolution image of reflectors in a platelike structure with a guided wave array of arbitrary shape is considered. The complete raw data set of signals from every transmitter-receiver combination is collected and then postprocessed. The general approach is to multiply the transmitted and received signals by suitable phase factors in order to focus the beam on every point within test structure. The algorithms differ in the way in which these phase coefficients are calculated.

Conventional technique is based on applying such phased shifts that all signals have equal phases at the focusing point. For an ultrasonic linear array this method is known as the Total Focusing Method [5]. In the current paper this method is referred to as the basicphased addition algorithm [4].

In principle the basic-phased addition algorithm can be applied to any array geometry, but its performance is good only for a limited number of cases. For example, it produces acceptable results for linear array [5], but in the case of a circular array a large number of elements is required to obtain acceptable resolution [4].

This paper is concerned with finding optimal phase coefficients, which allow the best resolution to be achieved for any array geometry with the minimum number of array elements. There is no requirement for the array elements to be omni-directional, i.e. to have equal transmission and reception sensitivity in all directions.

In order to obtain better resolution and higher sensitivity it is necessary to use higher order guided wave modes at higher frequencies, where multiple modes exist. Generalization of the above algorithms for the case of multimode media is suggested.

\section{Array and data acquisition}

An ultrasonic guided wave array system on an isotropic plate structure is considered. The array elements behave as point sources, $n_{T}$ elements function as transmitters and $n_{R}$ elements function as receivers. The time traces from each transmitter-receiver combination 
are collected and converted to frequency, $\omega$, domain. The complex spectrum of $n$-th signal is denoted by $s_{n}(\omega), n=1 . . N$, where $N=n_{T} n_{R}$ is the total number of transmitter receiver pairs in the array.

The wave field from any transmitter propagates into the plate, interacts with the scatterers and generates a scattered wave field. It is assumed that there is only one wave mode. Then, by using Born approximation, the scattered signals $s_{n}(\omega)$ can be written in the following form

$$
s_{n}(\omega)=\int K_{n}(\mathbf{r}, \omega) O(\mathbf{r}, \omega) d \mathbf{r},
$$

where $O(\mathbf{r}, \omega)$ is the unknown object distribution function characterizing the reflectors. Function $K_{n}(\mathbf{r}, \omega)$ for $\mathrm{i}$-th transmitter and $\mathrm{j}$-th receiver has the form:

$$
K_{n}(\mathbf{r}, \omega)=A(\omega) G\left(\mathbf{R}_{i}^{t}, \mathbf{r}, \omega\right) G\left(\mathbf{R}_{j}^{r}, \mathbf{r}, \omega\right),
$$

where $A(\omega)$ is the source excitation pulse, $G\left(\mathbf{r}^{\prime}, \mathbf{r}, \omega\right)$ is the Green's function of the structure, $\mathbf{R}_{i}^{t}$ is the location of the $\mathrm{i}$-th transmitter and $\mathbf{R}_{i}^{r}$ is the location of $\mathrm{j}$-th receiver.

The solution to the problem (1) is non-unique and unstable, i.e. the problem (1) is ill-posed. In the next sections the ways to obtain the stable approximation, which is "close" to the exact solution $O(\mathbf{r}, \omega)$ are discussed.

\section{Phased methods}

\subsection{Formulation}

The general idea of array processing is to multiply the signals $s_{n}(\omega)$ by suitable phase factors $t_{n}\left(\mathbf{r}_{0}, \omega\right)$ in order to focus the beam on every point $\mathbf{r}_{0}$ within test structure:

$$
O^{(1)}\left(\mathbf{r}_{0}, \omega\right) \equiv \sum_{n} t_{n}\left(\mathbf{r}_{0}, \omega\right) s_{n}(\omega),
$$

here $O^{(1)}\left(\mathbf{r}_{0}, \omega\right)$ is the approximation to the function $O\left(\mathbf{r}_{0}, \omega\right)$. Using equation (1), the functions $O^{(1)}\left(\mathbf{r}_{0}, \omega\right)$ and $O\left(\mathbf{r}_{0}, \omega\right)$ can be related by

$$
O^{(1)}\left(\mathbf{r}_{0}, \omega\right)=\int P\left(\mathbf{r}_{0}, \mathbf{r}, \omega\right) O(\mathbf{r}, \omega) d \mathbf{r}, \quad P\left(\mathbf{r}_{0}, \mathbf{r}, \omega\right) \equiv \sum_{n} t_{n}\left(\mathbf{r}_{0}, \omega\right) \mathrm{K}_{n}(\mathbf{r}, \omega) .
$$

Function $P\left(\mathbf{r}_{0}, \mathbf{r}, \omega\right)$ is the result for a point reflector located at $\mathbf{r}_{0}$ or the point spread function (PSF).

The results for all frequencies are then summed to synthesise the image:

$$
I\left(\mathbf{r}_{0}\right)=\left|\sum_{\omega} \frac{O^{(1)}\left(\mathbf{r}_{0}, \omega\right)}{P\left(\mathbf{r}_{0}, \mathbf{r}_{0}, \omega\right)}\right| .
$$

The processing algorithms differ in the way in which the phase coefficients $\mathbf{t}\left(\mathbf{r}_{0}, \omega\right)=\left\{t_{1}, . ., t_{N}\right\}^{T}$ are calculated.

\subsection{Basic-phased addition algorithm}

In the basic-phased addition method the coefficients are chosen to maximise the PSF at the point $\mathbf{r}_{0}$ in the image: 


$$
\frac{\left|P\left(\mathbf{r}_{0}, \mathbf{r}_{0}\right)\right|^{2}}{\left|\mathbf{t}\left(\mathbf{r}_{0}\right)\right|^{2}} \rightarrow \max
$$

\subsection{Method of maximization of contrast}

Another way of calculation the phase coefficient is to maximise the amplitude of the PSF at the focusing point, $\mathbf{r}_{0}$, relative to the amplitude of the PSF at all other points:

$$
\frac{\left|P\left(\mathbf{r}_{0}, \mathbf{r}_{0}\right)\right|^{2}}{\int\left|P\left(\mathbf{r}_{0}, \mathbf{r}\right)\right|^{2} \mathrm{~d} \mathbf{r}} \rightarrow \max
$$

This approach was firstly presented for the problem of focusing of bulk waves by a group of surface sources in [6].

\subsection{Least squares method}

By replacing the integral in (1) by a sum at discrete points $\mathbf{r}_{1}, . ., \mathbf{r}_{M}$, we get a system of linear equations

$$
\mathbf{K} \mathbf{x}=\mathbf{s}
$$

where $\mathbf{x}=\left\{O\left(\mathbf{r}_{1}\right), \ldots, O\left(\mathbf{r}_{M}\right)\right\}^{T}, \mathbf{K}=\left\{K_{n m}\right\}=\left\{K_{n}\left(\mathbf{r}_{m}\right)\right\}, \mathbf{s}=\left\{s_{1}, \ldots, s_{N}\right\}^{T}$.

To solve the system (8) we can use the Tikhonov regularization method [7]. Then (8) is replaced by the Tikhonov equation:

$$
\left(\alpha \mathbf{E}+\beta \mathbf{K}^{+} \mathbf{K}\right) \mathbf{x}^{(1)}=\mathbf{K}^{+} \mathbf{s},
$$

where $\mathbf{K}^{+}=\mathbf{K}^{* T}, \mathbf{E}$ is the identity matrix, and $\alpha$ and $\beta$ are regularization parameters.

If $\beta=0$ then equation (9) represents the Moore-Penrose pseudoinversion method. It can be shown that this is equivalent to the basic-phased addition algorithm (6). Therefore, the discrete PSF $\mathbf{P}=\left\{P_{n m}\right\}=\left\{P\left(\mathbf{r}_{n}, \mathbf{r}_{m}\right)\right\}$ and the approximate solution $\mathbf{x}^{(1)}$ for the basicphased addition method can be written in the form:

$$
\mathbf{P}=\mathbf{K}^{+} \mathbf{K}, \quad \mathbf{x}^{(1)}=\mathbf{K}^{+} \mathbf{s}
$$

If $\alpha=0$ then equation (9) represents the least squares method and it is possible to show that this is equivalent to the method of maximization of contrast (7). But in this case the solution is very unstable. To make it stable $\alpha=\alpha_{\text {opt }}>0$ is taken. Therefore, the PSF and the approximate solution for the method of maximization of contrast are given by:

$$
\mathbf{P}=\left(\alpha_{\text {opt }} \mathbf{E}+\mathbf{K}^{+} \mathbf{K}\right)^{-1} \mathbf{K}^{+} \mathbf{K}, \quad \mathbf{x}^{(1)}=\left(\alpha_{\text {opt }} \mathbf{E}+\mathbf{K}^{+} \mathbf{K}\right)^{-1} \mathbf{K}^{+} \mathbf{s}
$$

\subsection{Examples}

\subsubsection{Far-field approximation}

Consider the two dimensional problem when a point reflector is in the far field of an array. A polar coordinate system where $r$ and $\varphi$ represent, respectively, radial and angular position is defined, with its origin at the nominal centre of the array. In this case PSF can be written as

$$
P\left(r_{0}, r, \varphi_{0}, \varphi, \omega\right)=e^{i 2 k\left(r-r_{0}\right)} P_{\infty}\left(\varphi_{0}, \varphi, \omega\right),
$$

where $k=2 \pi / \lambda$ is wave number, $\lambda$ is wavelength and $P_{\infty}$ is angular PSF. 


\subsubsection{Linear array}

Fig.1(a) shows the angular PSF for a linear array with omni-directional point elements. It can be seen that in this case the basic-phased addition method gives better resolution than the method of maximization of contrast.

\subsubsection{Circular array}

Fig.1(b) shows the angular PSF for a circular array with omni-directional point elements. It can be seen that in this case, on the contrary, the method of maximization of contrast gives better resolution than basic-phased addition method.

Thus, the performance of each method depends on the geometry of the array.

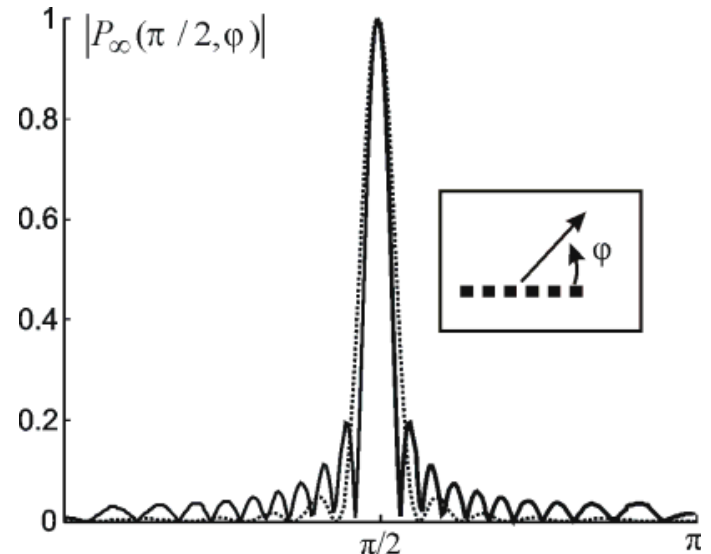

(a)

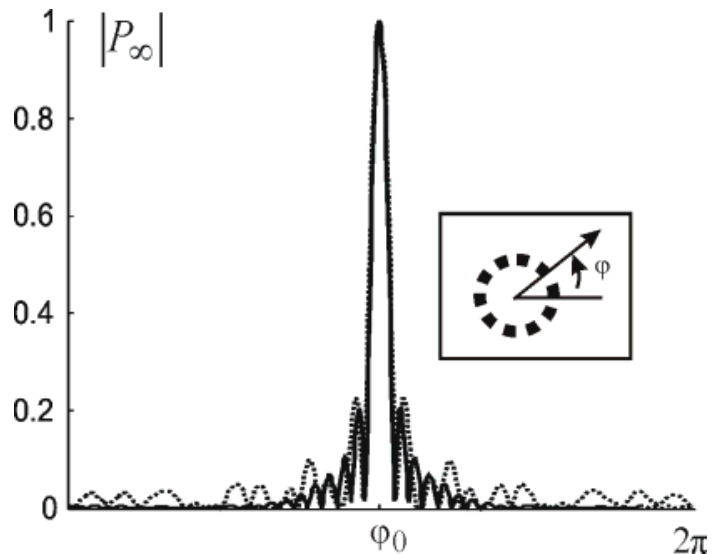

(b)

Fig. 1. Amplitude of PSF for (a) linear array, (b) circular array. Dotted line - basic-phased addition method, solid line - method of maximization of contrast.

\section{Improved processing}

\subsection{Deconvolution}

The expression (4) can be regarded as new equation for unknown function $O\left(\mathbf{r}_{0}, \omega\right)$. The question is: is it possible to improve the resolution given by the first approximation $O^{(1)}$ ?

Denote the angular part of $O^{(1)}$ in the case of far field by $x^{(1)}(\varphi, \omega)$. Then equation (4) can be written in the form

$$
x^{(1)}\left(\varphi_{0}, \omega\right)=\int_{0}^{2 \pi} P_{\infty}\left(\varphi_{0}, \varphi, \omega\right) x(\varphi, \omega) d \varphi,
$$

where $x(\varphi, \omega)=\int O(r, \varphi, \omega) e^{i 2 k\left(r-r_{0}\right)} r d r$.

For an axisymmetric array (for example the circular array shown in Fig.1(b)) equation (13) can be written as a relationship between Fourier coefficients:

$$
X^{(1)}(\Omega, \omega)=P_{\Omega}(\Omega, \omega) X(\Omega, \omega) \quad \Omega=1,2, \ldots
$$

Fig.2 shows the amplitude of the spectra $P_{\Omega}$ in $\Omega-\omega$ plane for the basic-phased addition algorithm and method of maximization of contrast. It can be seen that for both methods $P_{\Omega}(\Omega, \omega) \cong 0$ for $\Omega \geq \Omega_{0}(\omega) . \Omega_{0}$ describes the limit of angular resolution $\Delta \varphi$ of an array and depends only on the ratio of the diameter of an array to the wavelength. 
Using equation (14), the Fourier spectra for the second approximation can be written in the form [2]:

$$
X^{(2)}(\Omega, \omega)=F(\Omega, \omega)\left[P_{\Omega}(\Omega, \omega)\right]^{-1} X^{(1)}(\Omega, \omega),
$$

where $F(\Omega, \omega)=\exp \left\{-\Omega^{2} /\left(k^{2}(\omega) \gamma^{2}\right)\right\}$ is a Gaussian shaped filter function which suppresses data outside the region $\Omega \leq \Omega_{0}(\omega)$ and smoothes the resulting function in angular domain, $\gamma$ is a parameter defines the width of the filter.

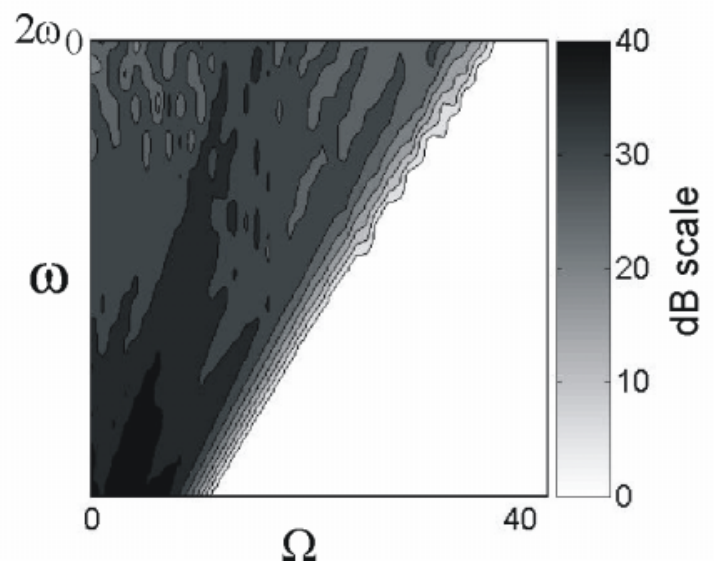

(a)

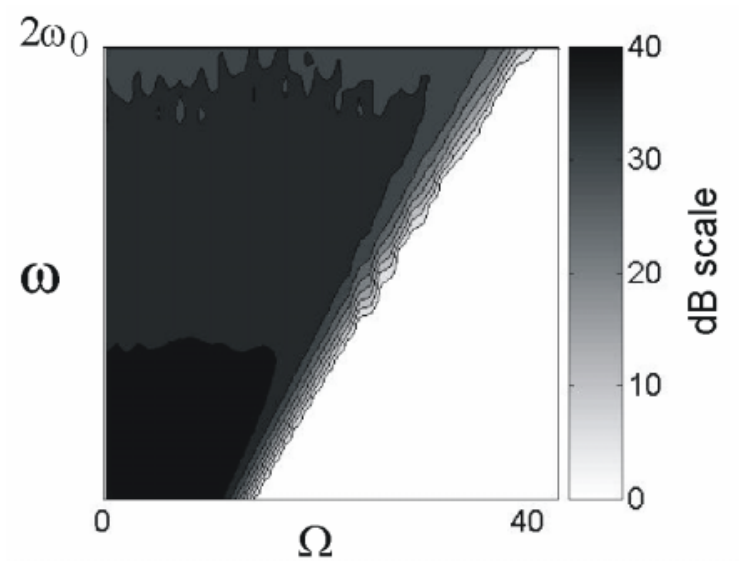

(b)

Fig. 2. Amplitude of the spectra $P_{\Omega}$ for (a) basic-phased addition method; (b) method of maximization of contrast.

\subsection{Maximization of contrast in the interval}

As have been shown each array has resolution limit characterised by some minimum spatial interval which can be resolved. The idea of the method of maximization of contrast in the interval is to maximise the PSF in this interval rather then in one focusing point as in (7). For far field resolution it can be written in the form:

$$
\frac{\int_{\varphi_{0}-\Delta \varphi}^{\varphi_{0}-\Delta \varphi}\left|P_{\infty}\left(\varphi_{0}, \varphi\right)\right|^{2} \mathrm{~d} \varphi}{\int_{0}^{2 \pi}\left|P_{\infty}\left(\varphi_{0}, \varphi\right)\right|^{2} \mathrm{~d} \varphi} \rightarrow \max
$$

Fig. 3 shows that maximization of contrast in the interval gives better resolution than previous methods for both linear and circular types of array. Moreover, in the case of a circular array the distribution of the amplitudes of the spectra $P_{\Omega}(\Omega, \omega)$ and filter function $F(\Omega, \omega)$ are very close to each other. This means that deconvolution does not improve the resolution very much in this situation. Thus, in practice the method of maximization of contrast in the interval gives the best possible resolution.

Note, that there is a maximum interelement spacing requirement in an array that must be adhered to in order to prevent the appearance of grating lobes. This maximum spacing does not depend on the method of array processing and it is approximately equal to $\lambda_{\min } / 2$, where $\lambda_{\min }$ is the shortest wavelength of guided waves within the frequency range of the transmitted signal. 


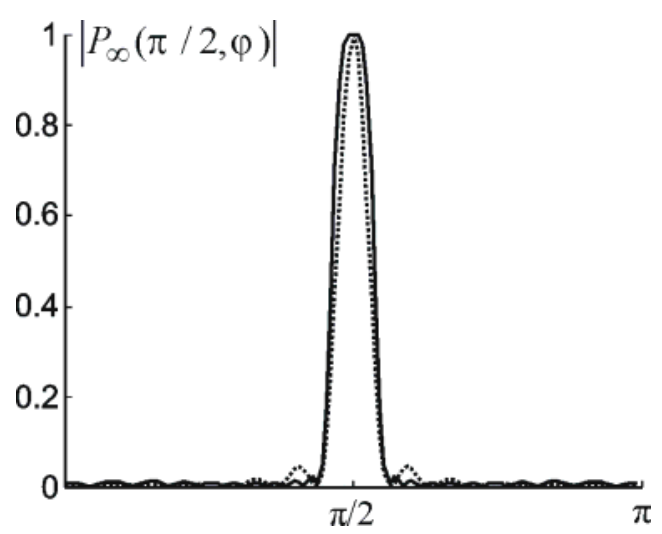

(a)

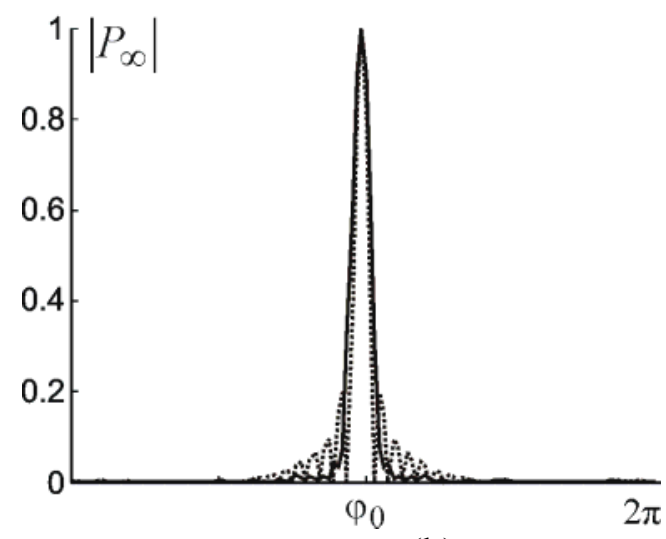

(b)

Fig.3. Amplitude of the PSF for (a) linear array; dotted line - basic-phased addition method; (b) circular array; dotted line - method of maximization of contrast in one direction. Solid line on both (a) and (b) - method of maximization of contrast in the interval.

\section{Results}

The experimental example considered is an array containing 16 transmitter elements and 32 receiver elements arranged in concentric rings with pitch circle diameters of 52 and 136 $\mathrm{mm}$, as shown in Fig.4. The array elements are EMATs designed to excite and detect the So Lamb wave mode and have equal transmission and reception sensitivity in all directions. The array was used on a $1.05 \mathrm{~m}$ by $1.25 \mathrm{~m}$ by $5-\mathrm{mm}$ thick aluminium plate specimen with square cut edges and 2-mm thick steel disk was bonded to the surface of the plate at the location shown to simulate a defect. The more detailed description of the experimental setup and array elements can be found in [2].

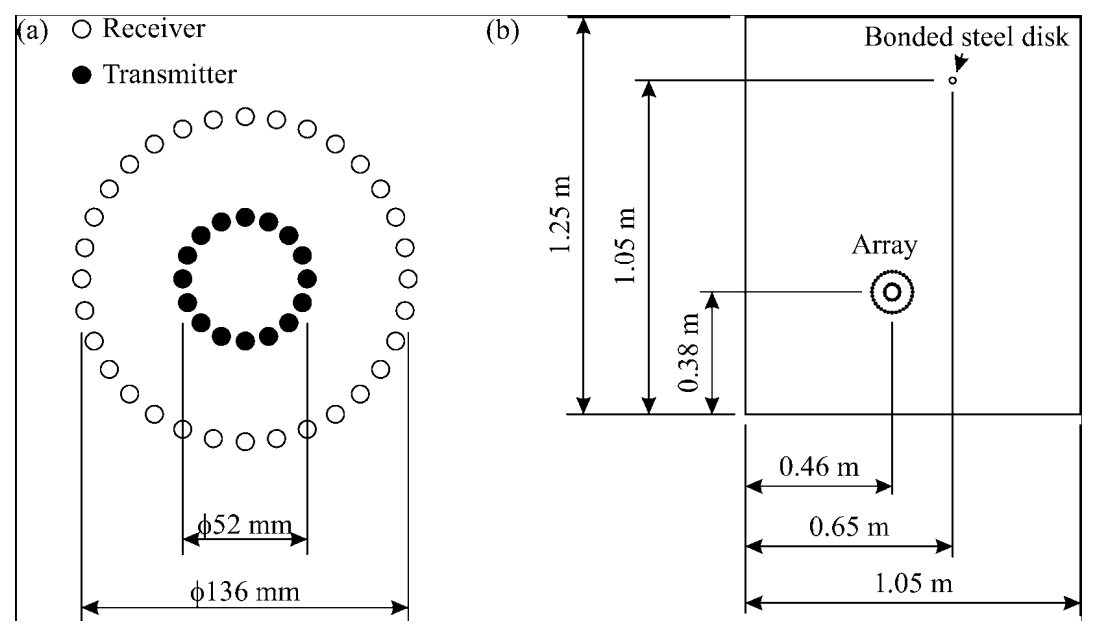

Fig.4. a) Geometry of EMAT array;

b) experimental arrangement on 5-mm thick aluminium plate specimen.

The results of data processing are shown in Fig.5. From Fig.5(a) and (b) it is seen that the basic-phased addition method and the method of maximization of contrast give many angular side-lobes. These side-lobes can be suppressed by deconvolution, the result obtained using the method of maximization of contrast with deconvolution is shown in Fig.5(c) (the phased addition algorithm with deconvolution gives the same resolution). Fig.5(d) shows that method of maximization of contrast in the interval gives approximately 
the same resolution as previous methods with deconvolution. This demonstrates the practical application of the technique to real experimental data.

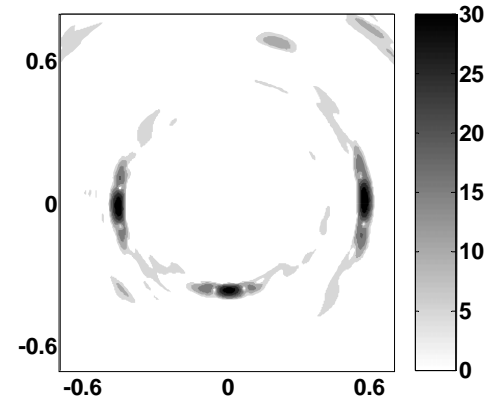

(a)

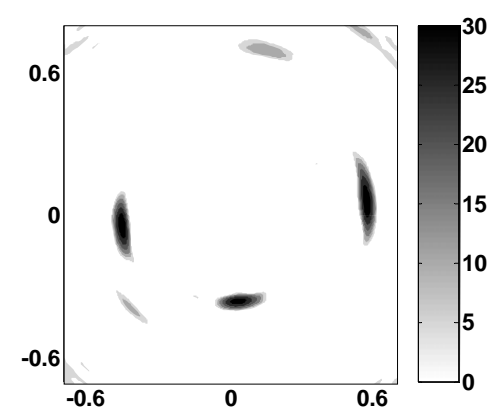

(c)

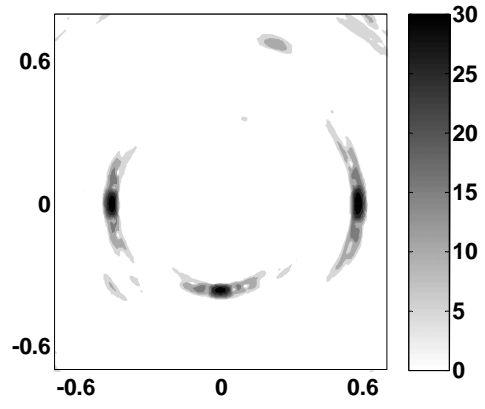

(b)

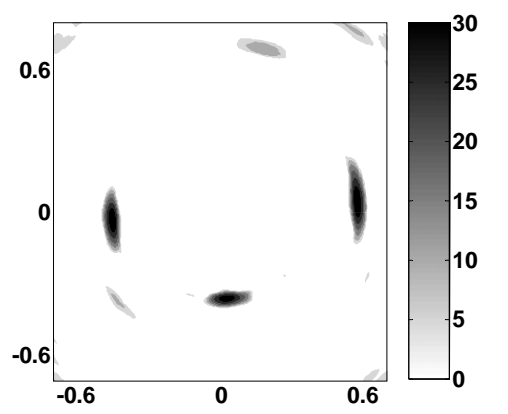

(d)

Fig.5. Results obtained using (a) basic phased addition algorithm, (b) method of maximization of contrast, (c) method of maximization of contrast with deconvolution, (d) method of maximization of contrast in the interval.

\section{Multimode resolution}

\subsection{Algorithm}

The processing algorithms described above can be generalised for the multimode media. But deconvolution in this case becomes quite complicated because instead of the scalar equation (13) there will be a system of linear equations. Therefore for multimode media the maximization of contrast in the interval seems to be the most appropriate method.

If there are several modes the problem of maximization (15) can be written for every transmitted-received mode combination:

$$
\frac{\int_{\varphi_{0}-\Delta \varphi_{m}}^{\varphi_{0}-\Delta \varphi_{m}}\left|P_{\infty}^{m}\left(\varphi_{0}, \varphi\right)\right|^{2} \mathrm{~d} \varphi}{\sum_{l}^{2 \pi} \int_{0}^{2 \pi}\left|P_{\infty}^{l}\left(\varphi_{0}, \varphi\right)\right|^{2} \mathrm{~d} \varphi} \rightarrow \max ,
$$

here index $m$ refers to the mode combination of interest and index $l$ refers to all possible mode combinations.

This method can be characterised by the set of point spread functions $\left\{P_{m n}\left(\varphi_{0}, \varphi\right)\right\}$. Each function $P_{m n}\left(\varphi_{0}, \varphi\right)$ describes the response from a point reflector for the $\mathrm{n}$-th mode combination if the extracted mode combination is the $\mathrm{m}$-th. For the case of ideal resolution $P_{m n}\left(\varphi_{0}, \varphi\right)=\delta_{m n} \delta\left(\varphi_{0}-\varphi\right)$. 


\subsection{Example}

The same configuration of array as in section 5 is considered. The inner ring has diameter $40 \mathrm{~mm}$ and contains 16 transducers, the outer ring has diameter $80 \mathrm{~mm}$ and contains 32 transducers. Each transducer operates as omni-directional transmitter and receiver. It is supposed that there are three modes in the system, $\mathrm{S}_{0}, \mathrm{~A}_{0}$ and $\mathrm{SH}_{0}$ and transducers are equally sensitive to all three modes. Fig. 6 shows the set of PSF for the case of $\mathrm{SH}_{0}-\mathrm{SH}_{0}$ mode combination extraction. The scale is logarithmic, so $10^{-2}$ corresponds to $-40 \mathrm{~dB}$ level.
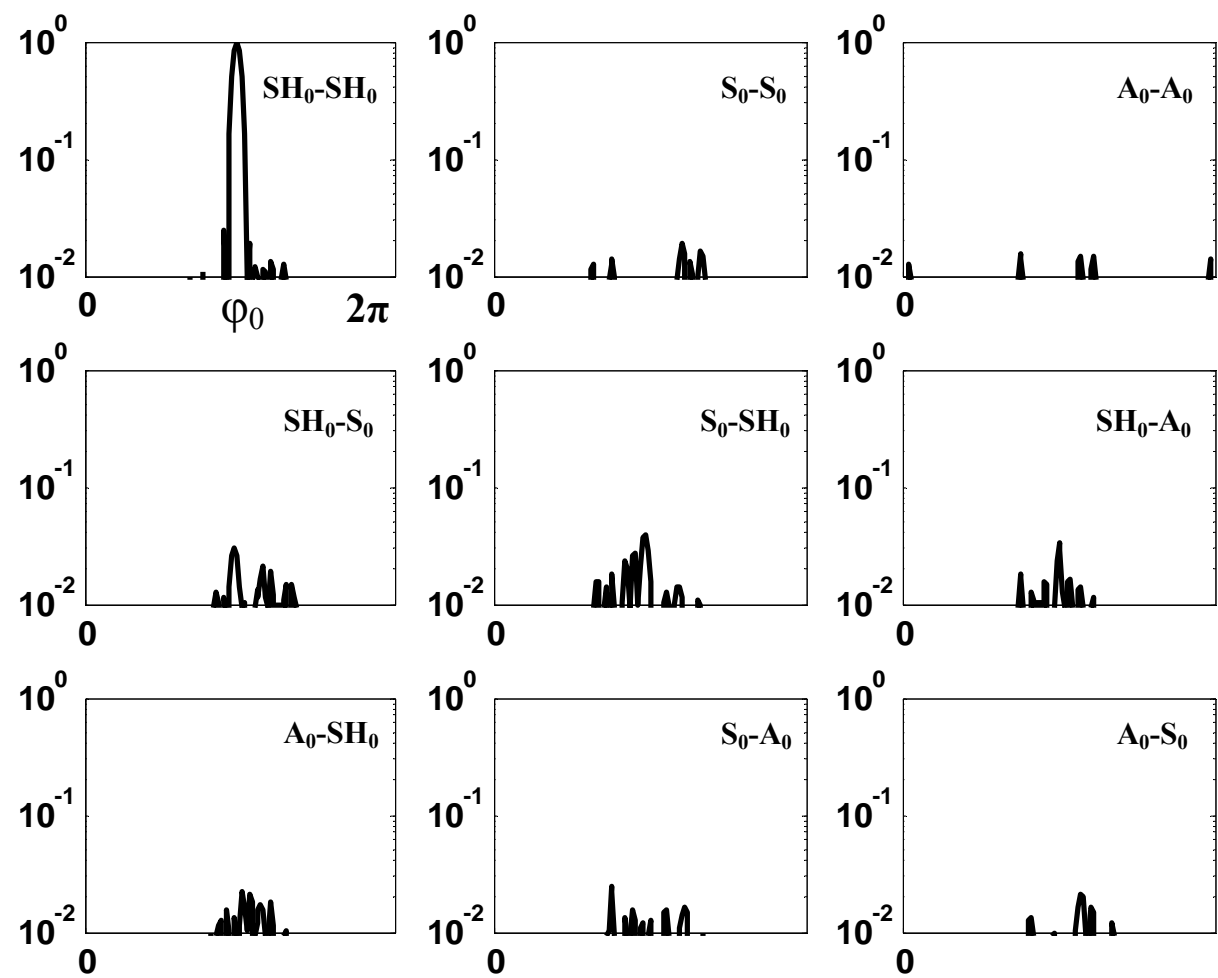

Fig.6. Amplitude of point spread functions for different mode combinations in the case of $\mathrm{SH}_{0}-\mathrm{SH}_{0}$ mode extraction; logarithmic scale.

\section{Conclusion}

A general procedure for array processing has been presented. The approach is based on multiplying the transmitted and received signals by suitable phase factors in order to focus the beam on every point within a test structure. The technique is applicable to any geometry of array and any type of array elements.

Different methods of calculating the phase coefficients have been considered. The performance of different algorithms has been tested on modelling and experimental data. It is shown that the method of maximization of contrast in the interval gives the best resolution.

Generalization of the above algorithms for the case of multimode media has been suggested. 


\section{Acknowledgement}

This work was supported by the UK Engineering and Physical Sciences Research Council (EPSRC) through the UK Research Centre in NDE and by BNFL, Nexia Solutions and DSTL.

\section{References}

[1] D. E. Chimenti, Guided waves in plates and their use in materials characterization, Appl. Mech. Rev., vol. 50, pp. 247-284, 1997.

[2] D. N. Alleyne, B. Pavlakovic, M. J. S. Lowe, and P. Cawley, Rapid long-range inspection of chemical plant pipework using guided waves, Insight, vol. 43, no. 2, pp. 93-96 and 101, 2001.

[3] R. Sicard, J. Goyette, and D. Zellouf, A SAFT algorithm for Lamb wave imaging of isotropic plate-like structures, Ultrasonics, vol. 39, pp. 487-494, 2002.

[4] P.D. Wilcox, Omni-directional guided wave transducer arrays for the rapid inspection of large areas of plate structures, IEEE Trans. on Ultrason., Ferroelect., Freq. Contr., vol. 50, no.6, pp. 699-709,2003.

[5] C. Holmes, B. Drinkwater and P. Wilcox, Post-processing of the full matrix of ultrasonic transmit-receive array data for non-destructive evaluation, NDT and E International, vol. 38, pp. 701-711, 2005.

[6] Babeshko V. A., Glushkov E. V., and Zinchenko J. F., The dynamics of inhomogeneous linearly-elastic bodies, M.: Nauka, 1989 (in Russian).

[7] A.N. Tikhonov, V.Y. Arsenin, Solutions of ill-posed problems, WH Winston, Washington DC, 1977. 